\title{
APPLICATION OF NUCLEAR REACTION ANALYSIS TO STUDYING HYDROGEN DIFFUSION AND NONSTOICHIOMETRY IN METAL OXIDES
}

\author{
V. B. Vykhodets ${ }^{1}$, T. E. Kurennykh ${ }^{1}$, O. A. Nefedova ${ }^{2} *$ \\ ${ }^{1}$ M.N. Miheev Institute of Metal Physics of Ural Branch of Russian Academy of Sciences, 18 S. Kovalevskoy st., \\ Ekaterinburg, Russian Federation \\ ${ }^{2}$ Institute of Engineering Science, Ural Branch of the Russian Academy of Sciences, 34 Komsomolskaya st., \\ Ekaterinburg, Russian Federation
}

*Corresponding author. E-mail: nefedova@imach.uran.ru; address for correspondence: 34, ul. Komsomolskaya, 620049, Ekaterinburg, Russian Federation. Tel.: +7 343 3753592; fax: +7 3433745330

Nuclear reaction analysis is used to investigate hydrogen and oxygen subsystems for the oxides $\mathrm{TiO}_{2}, \mathrm{La}_{0,9} \mathrm{Sr}_{0,1} \mathrm{ScO}_{3-\delta}$ and $\mathrm{ZrO}_{2}\left(10 \% \mathrm{Y}_{2} \mathrm{O}_{3}\right)$. For comparison, data on deuterium diffusion in $\mathrm{Ni}$ are obtained. It is found that the boundary conditions of the diffusion problem for the metal differ greatly from those for the oxides. The general and partial solutions to the diffusion problem have been obtained for this technique. It is shown that, for metals, the experimental data correspond to the condition of the zero flow of deuterium atoms through the irradiated surface, whereas for oxides, these data correspond to the zero concentration of deuterium on the surface. It is found that the atomic surface layer of nanoparticles of titanium dioxide and yttrium-doped zirconium dioxide lacks oxygen in comparison with stoichiometry. Nanopowder synthesis is performed by laser evaporation of a ceramic target. The injection of the $\mathrm{D}_{2} \mathrm{O}$ vapor into the working chamber does not lead to the doping of nanoparticles with deuterium and the elimination of oxygen deficiency.

Keywords: hydrogen diffusion, oxygen stoichiometry, nuclear reaction analysis, oxides, nickel, diffusion boundary value problem with an internal source, analytical solution.

DOI: $10.17804 / 2410-9908.2015 .5 .031-044$

\section{References}

1. Fishman A., Kurennykh T., Vykhodets V., Vykhodets E. Oxygen Isotope Exchange in Nanocrystal Oxides. In: Advances in Ceramics-Characterization, Raw Materials, Processing, Properties, Degradation and Healing, C. Sikalidis, ed. Rejeka: InTechOpen Access publisher, 2011, pp. 139-164. ISBN 978-953-307-504-4.

2. Kudo H., Kosaku Y., Ando Y., Hiraga M., Sekine T. Deuterium migration in titanium during deuteron irradiation observed by proton spectra of the $\mathrm{d}(\mathrm{d}, \mathrm{p}) \mathrm{t}$ reaction. Journal of Nuclear Materials, 1998, vols 258-263, p. 1, pp. 622-627.

3. Vykhodets V.B., Kurennykh T.E., Nefedova O.A., Gorelov V.P., Stroeva A.Yu., Balakireva V.B., Vykhodets E.V., Obukhov S.I. Deuterium diffusion in proton conductors $\mathrm{La}_{0.9} \mathrm{Sr}_{0.1} \mathrm{ScO}_{3-\delta}$ and $\mathrm{BaZr}_{0.9} \mathrm{Y}_{0.1} \mathrm{O}_{3-\delta}$ at room temperature. Solid State Ionics, 2014, vol. 263, pp. 152-156. DOI: 10.1016/j.ssi.2014.06.003.

4. Kidson G.V. The diffusion of H, D, and T in solid metals. Diffusion in Solid Metals and Alloys, vol. III-26, H. Mehrer, ed. Berlin, Landolt-Bornstein, Springer-Verlag Publ., 1990, p. 504. ISBN 3-54050-886-4.

5. Völk1 J., Alefeld G. Hydrogen Diffusion in Metals. Diffusion in Solids: Recent Developments, A.S. Nowick, ed. J.J. Burton. New York, Academic Press Publ., 1975, pp. 231-302. ISBN 0-12-522660-8.

6. Heitjans P., Kärger J., eds. Diffusion in Condensed Matter: Methods, Materials, Models. Berlin-Heidelberg, Springer Publ., 2005, 965 p. ISBN 3-540-20043-6. 
open-access journal

7. Vykhodets V.B., Jarvis E., Kurennykh T.E., Davletshin A.E., Obukhov S.I., Beketov I.V., Samatov O.M., Medvedev A.I. Extreme deviations from stoichiometry in alumina nanopowders. Surface Science, 2014, vol. 630, pp. 182-186. DOI: 10.1016/j.susc.2014.08.009.

8. Jarvis E.A.A., Carter E.A. Metallic Character of the $\mathrm{Al}_{2} \mathrm{O}_{3}(0001)-(\sqrt{31} \times \sqrt{31}) \mathrm{R} \pm 9^{\circ}$ Surface Reconstruction. J. Phys. Chem. B, 2001, vol. 105, iss. 18, pp. 4045-4052. DOI: 10.1021/jp003587c.

9. Wang X.-G., Chaka A., Scheffer M. Effect of the Environment on a- $\mathrm{Al}_{2} \mathrm{O}_{3}(0001)$ Surface Structures. Physical Review Letters, 2000, vol. 84, pp. 3650-3653.

10. Crank J. The mathematics of diffusion. London, Oxford University Press Publ., 1975, 414 p. ISBN 0-19-853344-6.

11. Nellis G., Klein S. Heat Transfer. Cambridge, Cambridge University Press Publ., 2009, 1107 p. ISBN 978-0-521-88107-4.

12. Kartashov E.M. Analiticheskie metody v teorii teploprovodnosti tverdykh tel [Analytical Methods in the Theory of Heat Conduction of Solids]. M., Vysshaya Shkola Publ., 2001, 550 p. (In Russian). 
Подана в журнал: 25.09.2015

УДК 544.6:544.16:539.2:517.958

DOI: $10.17804 / 2410-9908.2015 .5 .031-044$

\title{
ПРИМЕНЕНИЕ ЯДЕРНОГО МИКРОАНАЛИЗА ДЛЯ ИССЛЕДОВАНИЯ ДИФФУЗИИ ВОДОРОДА И НЕСТЕХИОМЕТРИИ В ОКСИДАХ МЕТАЛЛОВ
}

\author{
В. Б. Выходец ${ }^{1}$, Т. Е. Куренных ${ }^{1}$, О. А. Нефедова ${ }^{2} *$ \\ ${ }^{1}$ Федеральное государственное бюджетное учреждение науки Институт физики металлов имени \\ М.Н. Михеева Уральского отделения Российской академии наук, ул. С. Ковалевской, 18, Екатеринбург, \\ Российская Федерачия \\ ${ }^{2}$ Федеральное государственное бюджетное учреждение науки Институт машиноведения Уральского \\ отделения Российской академии наук, ул. Комсомольская, 34, Екатеринбург, Российская Федерация
}

*Ответственный автор. Электронная почта: nefedova@imach.uran.ru; адрес для переписки: ул. Комсомольская, 34, Екатеринбург, Российская Федерация. Телефон: +7 (343) 375-35-92; факс: +7 (343) 374-53-30

Ядерный микроанализ применен для исследования водородной и кислородной подсистем оксидов: $\mathrm{TiO}_{2}, \mathrm{La}_{0,9} \mathrm{Sr}_{0,1} \mathrm{ScO}_{3-\delta}$ и $\mathrm{ZrO}_{2}\left(10 \% \mathrm{Y}_{2} \mathrm{O}_{3}\right)$. Для сравнения получены данные по диффузии дейтерия в Ni. Установлено, что для металла и оксида реализуются сильно отличающиеся друг от друга граничные условия диффузионной задачи. Получены общее и частные решения диффузионного уравнения для этой методики. Обнаружено, что для металла экспериментальные данные соответствуют условию нулевого потока атомов дейтерия через облучаемую поверхность, а для оксида - нулевой концентрации дейтерия на этой поверхности. Получено, что в поверхностном атомном слое наночастиц диоксида титана и диоксида циркония, допированного иттрием, имеет место дефицит кислорода в сравнении со стехиометрией. Синтез нанопорошков осуществлялся с помощью технологии лазерного испарения керамической мишени. Введение пара $\mathrm{D}_{2} \mathrm{O}$ в рабочую камеру при синтезе нанопорошков диоксида титана не приводило к легированию наночастиц дейтерием и к ликвидации кислородного дефицита.

Ключевые слова: диффузия водорода, кислородная нестехиометрия, ядерный микроанализ, оксиды, никель, краевая задача диффузии с внутренним источником, аналитическое решение.

\section{1. Введение}

Оксиды металлов являются перспективными функциональными материалами для катализа, медицины, ядерной и водородной энергетики и других приложений. Важными характеристиками этих материалов являются данные об их химическом составе, в частности отклонения от стехиометрии, а также сведения о взаимодействии оксидов с водородом, включая информацию о растворимости и диффузии в них изотопов водорода. В последнее время было выполнено несколько работ, в которых для исследования водородной и кислородной подсистем оксидов была применена ускорительная методика ядерного микроанализа (NRA). C ее помощью были получены данные о кислородном и водородном изотопном обмене и коэффициентах диффузии атомов кислорода и дейтерия в оксидах [1-3]. Кроме того, для проведения исследований по поверхностной стехиометрии оксидов и диффузии в них дейтерия были разработаны две оригинальные методики, основанные на применении NRA. Они имеют значительные перспективы, и их дальнейшее развитие представляет интерес. В настоящей работе рассмотрены некоторые актуальные аспекты этих оригинальных методик.

Первая из них (NRAOL) [3] ориентирована на получение с помощью прямого метода данных по коэффициентам диффузии и может быть применена как для исследования 
диффузии дейтерия как в металлах, так и в оксидах. Она предполагает измерение коэффициентов диффузии в режиме реального времени (on-line). При ее применении производится непрерывное облучение образца дейтронами с энергией в несколько сот кэВ, при этом в образце формируется внутренний источник диффузии меченых атомов водорода (дейтерия), одновременно с этим в объекте исследования протекает диффузия атомов дейтерия и также одновременно осуществляется измерение концентрационного профиля атомов дейтерия с помощью ядерной реакции ${ }^{2} \mathrm{H}(\mathrm{d}, \mathrm{p})^{3} \mathrm{H}$. B рамках NRAOL, как и в случае классических методик, реализуется три экспериментальных этапа диффузионного эксперимента, а именно: создание источника диффузии меченых атомов, изотермический диффузионный отжиг образца и измерение концентрационного профиля, сформированного при отжиге. В то же время использование NRAOL обусловливает два важных ее преимущества перед классическими аналогами. Во-первых, проведение перечисленных этапов в режиме on-line позволяет проводить исследования в системах, в которых имеет место быстрая диффузия даже при комнатной температуре. Для водорода эта особенность NRAOL актуальна в связи с тем, что атомы водорода характеризуются наибольшей скоростью диффузии среди всех элементов. Во-вторых, в случае NRAOL диффузионные процессы протекают исключительно в объеме образца. В случае других методик серьезной проблемой является неконтролируемое влияние процессов на границе образца на кинетику проникновения или десорбции водорода, что обусловливает ненадежность многих существующих данных по коэффициентам диффузии водорода [4-6], особенно при низких температурах. В разработанном варианте методики NRAOL [3] при формулировании начальных и граничных условий диффузионной задачи постулировалось, что атомы дейтерия либо вообще не выходят из образца при его облучении дейтронами, либо это условие выполняется с достаточно высокой точностью в случае низких температур. Однако, позднее при применении методики NRAOL для исследования диффузии дейтерия в различных материалах было установлено, что ситуация с граничными и начальными условиями диффузионной задачи в методике NRAOL является более сложной, чем это предполагалось. В связи с этим в настоящей работе проведено экспериментальное и теоретическое исследование по этому вопросу, имеющее целью обеспечить корректное применение методики NRAOL.

Вторая оригинальная методика [7] ориентирована на получение данных о распределении ионов кислорода в оксидных наночастицах. Она предполагает синтез оксидных нанопорошков, кислородная подсистема которых обогащена мало распространенным изотопом ${ }^{18} \mathrm{O}$, и измерение с помощью NRA концентраций изотопов ${ }^{16} \mathrm{O}$ и ${ }^{18} \mathrm{O}$ в ансамбле наночастиц в зависимости от удельной поверхности нанопорошка. Полученные в работе [7] экспериментальные данные свидетельствовали о сильном дефиците кислорода по сравнению со стехиометрией в поверхностном атомном слое наночастиц оксида алюминия. Ранее такой же результат для массивного монокристалла оксида алюминия был получен из первых принципов с помощью теории функционала плотности [8], теоретические данные характеризовали оксид в состоянии термодинамического равновесия. В совокупности в упомянутых теоретических и экспериментальных работах развивались новые представления о структуре оксидов, они могут иметь большое значение для теории и практических приложений как для массивных материалов, так и для наноразмерных пленок и порошков. Важный результат по поверхностной стехиометрии в оксидах был получен также в теоретической работе [9], выполненной с помощью теории функционала плотности для оксида алюминия, где было показано, что легирование кристалла водородом должно приводить к сильному изменению поверхностной стехиометрии, конкретно - к ликвидации кислородного дефицита в поверхностном слое оксида. Этот результат может иметь большое значение для практических приложений, поскольку он указывает на принципиальную возможность получения оксидных нанопорошков с регулируемой поверхностной 
стехиометрией. Экспериментальных работ по этому вопросу нет, и в настоящей работе было проведено поисковое исследование по легированию оксидов водородом (дейтерием) и влиянию этого легирования на поверхностную стехиометрию в оксидных нанопорошках. Таким образом, основной задачей этой работы было получение данных о водородной и кислородной подсистемах оксидов металлов с помощью ядерного микроанализа, включая решение научных и методических вопросов.

\section{2. Методики и образцы}

Исследования с помощью методик NRA и NRAOL проводились на 2 MB ускорителе Ван де Граафа. Для измерения концентрационных профилей атомов дейтерия в образцах использовали реакцию ${ }^{2} \mathrm{H}(\mathrm{d}, \mathrm{p})^{3} \mathrm{H}$ при энергии частиц первичного пучка 650 кэВ. В аналогичных измерениях для изотопа кислорода ${ }^{18} \mathrm{O}$ использовали реакцию ${ }^{18} \mathrm{O}(\mathrm{p}, \alpha){ }^{15} \mathrm{~N}$ при энергии протонов 762 кэВ, а для изотопа ${ }^{16} \mathrm{O}$ - реакцию ${ }^{16} \mathrm{O}\left(\mathrm{d}, \mathrm{p}_{1}\right){ }^{17} \mathrm{O}^{*}$ при энергии дейтронов 900 кэВ. Диаметр первичных пучков дейтронов и протонов составлял от 0,5 до 1 мм. Дозу облучения образцов определяли с помощью вторичного монитора, статистическая погрешность в этих измерениях была около 0,5 \%. Для проведения ядерно-физических измерений на оксидных нанопорошках их частицы впрессовывались в индиевую пластину, эти исследования проводили при комнатной температуре. В случае массивных образцов керамик и никеля использовали специальный держатель, позволяющий производить нагрев и охлаждение образцов при их облучении дейтронами. Для нагрева и охлаждения образцов использовали термоэлектрические модули промышленного производства, основанные на эффекте Пельтье. Конструкция держателя позволяла проводить исследования в интервале температур от - 30 до $130{ }^{\circ} \mathrm{C}$, температуру образцов во время облучения измеряли с помощью резистивного датчика, колебания температуры при облучении образца не превышали $0,5^{\circ} \mathrm{C}$. В этом держателе надежный тепловой контакт между пластиной модуля Пельтье и образцом обеспечивался с помощью механических прижимов, индиевых и резиновых прокладок с высокой теплопроводностью. Когда требовалось провести измерения на оксидных нанопорошках с помощью NRA при более высоких температурах, для нагрева образца использовали резистивную печь сопротивления. В этом случае частицы оксида впрессовывали в медный порошок. Такой держатель позволял нагревать образец до $400{ }^{\circ} \mathrm{C}$.

Эксперименты по диффузии дейтерия с помощью методики NRAOL проводили на образцах никеля чистотой 99,99 \% и на плотной керамике оксида $\mathrm{La}_{0,9} \mathrm{Sr}_{0,1} \mathrm{ScO}_{3-\delta}$, она была синтезирована с помощью высокотемпературной керамической технологии из оксидов лантана, стронция и скандия. Эксперименты по поверхностной стехиометрии с помощью NRA и изотопных методов проводили на оксидных нанопорошках. Нанопорошки диоксида титана и диоксида циркония, допированного иттрием, были получены с помощью технологии лазерного испарения керамической мишени. Перед изготовлением мишеней кислородная подсистема исходных порошков была обогащена изотопом ${ }^{18} \mathrm{O}$. Для этого проводился отжиг исходных порошков в проточном кислороде, обогащенном на $80 \%$ изотопом ${ }^{18} \mathrm{O}$. Давление кислорода при отжигах было 1 атм. Температура отжига для диоксида титана составляла $1000{ }^{\circ} \mathrm{C}$, продолжительность 5 суток. Для диоксида циркония, допированного иттрием, соответствующие параметры были $800{ }^{\circ} \mathrm{C}$ и 24 ч. При синтезе нанопорошков использовался волоконный иттербиевый лазер с длиной волны $1,07 \mu \mathrm{m}$, максимальная мощность 1 кВт. Технология синтеза была ориентирована на получение нанопорошков как не содержащих, так и содержащих атомы водорода. Делалось это для того, чтобы получить данные о влиянии атомов водорода на поверхностную стехиометрию в оксидных наночастицах (см. разд. 1). В связи с этим, синтез нанопорошков диоксида титана проводили в двух отличающихся друг от друга средах: в инертных газах (гелии или аргоне) и в инертных газах, содержащих некоторое количество водяного пара. Он рассматривался нами как источник атомов водорода при легировании оксида водородом. В экспериментах

Vykhodets V.B. et al. / Application of nuclear reaction analysis to studying hydrogen diffusion and nonstoichiometry in metal oxides 
использовались насыщенные пары $\mathrm{D}_{2} \mathrm{O}$. Температура воды, находящейся в равновесии с паром, была $45{ }^{\circ} \mathrm{C}$. Использование тяжелой по водороду воды было обусловлено тем, что в этом случае с помощью реакции ${ }^{2} \mathrm{H}(\mathrm{d}, \mathrm{p})^{3} \mathrm{H}$ можно определить концентрацию дейтерия в нанопорошках. В случае диоксида циркония, допированного иттрием, синтез нанопорошков проводился только в среде инертных газов. Для получения нанопорошков с различным средним размером частиц в широком диапазоне значений удельной поверхности варьировали сорт инертного газа (аргон или гелий), его давление, мощность лазера, использовали как импульсный, так и непрерывный режим лазерного облучения мишени. Удельные поверхности порошков определяли с помощью БЭТ-методики. Более подробно условия экспериментов на ускорителе, синтеза плотной керамики и нанопорошков, а также процедур математической обработки данных ядерно-физических экспериментов изложены в работах $[3,7]$.

\section{3. Результаты и обсуждение}

\section{1 Граничные условия диффузионной задачи при использовании методики NRAOL}

Как отмечалось в разд. 1, в работе [3] для методики NRAOL использовалось граничное условие диффузионной задачи, согласно которому атомы дейтерия вообще не выходят из образца при его облучении дейтронами. Тем самым принимается существование достаточно высокого потенциального барьера для выхода в вакуум из образца ионов дейтерия. Основанием для применения указанного граничного условия в работе [3] были результаты предварительных экспериментов, которые проводились при комнатной температуре на микропорошках оксидов $\mathrm{La}_{0,9} \mathrm{Sr}_{0,1} \mathrm{ScO}_{3-\delta}$ и $\mathrm{BaZr}_{0,9} \mathrm{Y}_{0,1} \mathrm{O}_{3-\delta}$. В порошках сначала были растворены пары тяжелой воды $\mathrm{D}_{2} \mathrm{O}$, а затем с помощью методики NRA получены спектры продуктов ядерной реакции ${ }^{2} \mathrm{H}(\mathrm{d}, \mathrm{p})^{3} \mathrm{H}$. Они соответствовали образцам с постоянной по глубине концентрацией дейтерия, и в пределах погрешности эксперимента эти спектры не менялись для порошков, которые хранились различное время в вакууме или на воздухе. На основе этих результатов и было сформулировано упомянутое выше граничное условие диффузионной задачи. Из общих соображений можно было полагать, что это условие будет справедливо и в случае более низких температур. При выполнении настоящей работы аналогичный эксперимент был проведен при температуре около $100{ }^{\circ} \mathrm{C}$. В нем использовался микропорошок оксида $\mathrm{La}_{0,9} \mathrm{Sr}_{0,1} \mathrm{ScO}_{3-\delta}$, в котором были растворены пары $\mathrm{D}_{2} \mathrm{O}$. Сначала концентрация дейтерия была измерена при комнатной температуре, затем порошок был нагрет в камере ускорительной установки, и измерения концентрации дейтерия проводились в течение нескольких часов. Результат был такой же, что и при комнатной температуре в работе [3] - содержание дейтерия в порошках оставалось постоянным.

Эти результаты были использованы при формулировке граничных условий диффузионной задачи для методики NRAOL. Эксперименты в настоящей работе проводились на никеле и оксиде $\mathrm{La}_{0,9} \mathrm{Sr}_{0,1} \mathrm{ScO}_{3-\delta}$. На рис. 1 приведены выборочные спектры продуктов ядерной реакции ${ }^{2} \mathrm{H}(\mathrm{d}, \mathrm{p})^{3} \mathrm{H}$ для этих материалов. Для методики NRAOL легко подобрать параметры эксперимента, при которых форма спектра реакции ${ }^{2} \mathrm{H}(\mathrm{d}, \mathrm{p})^{3} \mathrm{H}$ будет соответствовать образцу с примерно постоянной по глубине концентрацией дейтерия в зоне анализа. Этими условиями являются достаточно высокие значения коэффициента диффузии дейтерия и времени облучения образца, а также выполнение граничного условия диффузионной задачи, о котором говорилось выше. В данной работе было получено несколько десятков спектров для образцов металла и оксида при различных температурах образцов, дозах и временах облучения. Для представления на рис. 1 были выбраны спектры, которые по времени облучения и ожидаемым значениям коэффициентов диффузии заведомо соответствовали условиям, необходимым для получения спектров, характерных для образцов с примерно постоянной по глубине концентрацией дейтерия. Однако из рис. 1 видно, что

Vykhodets V.B. et al. / Application of nuclear reaction analysis to studying hydrogen diffusion and nonstoichiometry in metal oxides 
спектры реакций для никеля и оксида кардинально различаются. Для металла спектр соответствует ожидаемому, а форма спектра для оксида свидетельствует о примерно нулевом значении концентрации дейтерия вблизи облучаемой поверхности образца и о невыполнении для оксида граничного условия диффузионной задачи, согласно которому поток атомов дейтерия через облучаемую поверхность

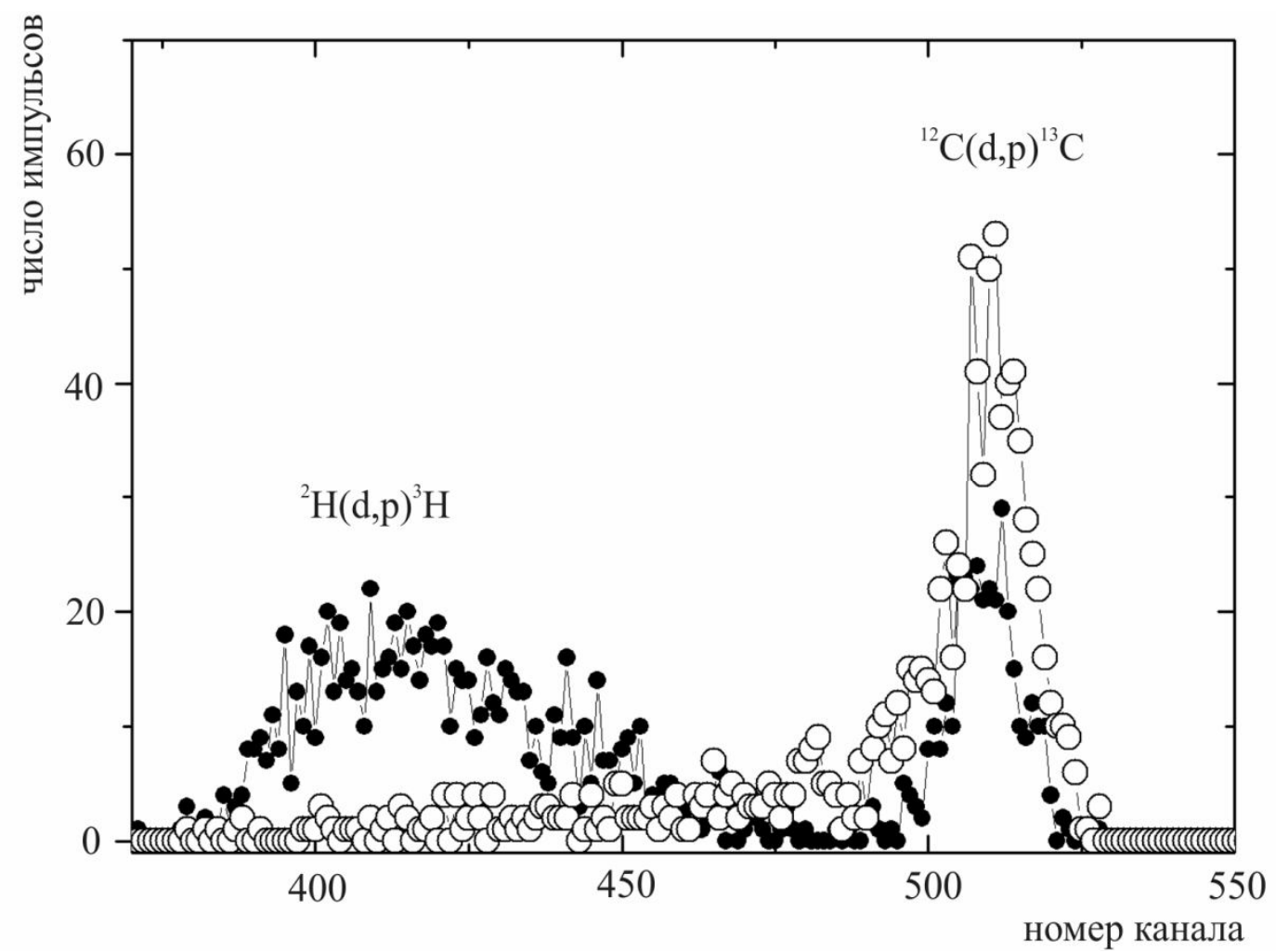

Рис. 1. Спектры продуктов ядерной реакции ${ }^{2} \mathrm{H}(\mathrm{d}, \mathrm{p})^{3} \mathrm{H}$ для образцов никеля (залитые кружки) и плотной керамики оксида $\mathrm{La}_{0,9} \mathrm{Sr}_{0,1} \mathrm{ScO}_{3-\delta}$ при одинаковой дозе облучения, температуре образцов $(276 \mathrm{~K})$ и времени облучения $(11300 \mathrm{c})$

образца равен нулю. Можно отметить, что этот результат не связан со структурой и температурой образцов оксида, он наблюдался во всем исследованном интервале температур как для плотной керамики, так и для микропорошка. Он также является неожиданным, если принять во внимание представленные выше данные о высоком потенциальном барьере для выхода атомов дейтерия из образцов оксидов, содержащих растворенные пары $\mathrm{D}_{2} \mathrm{O}$.

Физическая природа обнаруженного явления в данной работе не исследовалась. Можно предполагать, что этот эффект связан с нарушением условия зарядовой компенсации при облучении кристаллов оксида дейтронами. Другими словами, атомы дейтерия покидают образец оксида из-за того, что состояние ионного соединения с избыточным зарядом оказывается энергетически невыгодным. В случае же металла (никеля) происходит экранировка заряда имплантированного иона дейтерия свободными электронами, и при достаточно низких температурах имплантированные ионы дейтерия не выходят из образца в вакуум. Так или иначе, полученные здесь результаты показали необходимость модификации граничных условий диффузионной задачи в методике NRAOL. Ниже предлагается общий подход к решению этой задачи, ориентированный на исследование диффузии дейтерия как в металлах, так и в оксидах.

Рассмотрено решение одномерной краевой задачи диффузии для полуограниченного образца с постоянно действующим внутренним источником, расположенным на глубине $x=x_{0}$. 
Дифференциальное уравнение диффузионного процесса с внутренним источником, согласно [10], имеет вид:

$$
\frac{\partial c(x, t)}{\partial t}=D \frac{\partial^{2} c(x, t)}{\partial x^{2}}+f(x, t),(0<x<\infty, t>0) .
$$

Здесь $c(x, t)$ - функция распределения для концентрации дейтерия; $x$ - глубина в образце; $t-$ время облучения; функция $f(x, t)$ характеризует работу источника и задается в виде:

$$
f(x, t)=w \delta\left(x-x_{0}\right),
$$

где $w=I /\left(S q_{0}\right)$ - поток дейтронов, падающих на образец; $I$ - сила тока пучка дейтронов, падающих на образец; $S$ - площадь поперечного сечения пучка дейтронов; $q_{0}$ - заряд дейтрона; $\delta\left(x-x_{0}\right)$ - дельта-функция Дирака.

В начальный момент времени дейтерия в образце не было, следовательно

$$
c(x, 0)=0 .
$$

На правой границе для полуограниченного тела принимаем условие

$$
c(\infty, t)=0
$$

Вид граничных условий на левой границе определяется материалом образца. Для оксида наилучшему приближению экспериментальных данных соответствуют граничные условия первого рода в виде нулевой концентрации

$$
c(0, t)=0
$$

для металла принимаем граничное условие второго рода в виде отсутствия потока ионов дейтерия через поверхность образца

$$
\frac{\partial c(0, t)}{\partial x}=0
$$

Также были рассмотрены обобщающие граничные условия третьего рода

$$
\frac{\partial c(0, t)}{\partial x}-h c(0, t)=0 .
$$

Здесь $h$ - коэффициент пропорциональности. Из граничного условия третьего рода как частный случай, можно получить граничное условие первого рода (5) при $h \rightarrow \infty$. Аналогично при $h \rightarrow 0$ из (7) получаем граничное условие (6).

Для построения решения краевой задачи применялся метод функций Грина [11]. Сначала определялась функция Грина $G(x, \xi, t-\tau)$ - решение краевой задачи диффузии того же типа, но с мгновенным точечным источником. Затем источник диффузии исходной задачи рассматривался как совокупность мгновенных источников, и решение исходной задачи представлялось в интегральном виде:

$$
c(x, t)=\int_{0}^{t+\infty} \int_{0}^{+\infty} f(\xi, \tau) G(x, \xi, t-\tau) d \xi d \tau
$$


При решении каждой из задач (1)-(5); (1)-(4); (6) и (1)-(4); (7) были использованы следующие представления функции Грина для случая неограниченной прямой $(-\infty<x<+\infty)$, найденные в [12]:

- при граничных условиях вида (5)

$$
G(x, \xi, t-\tau)=\frac{1}{2 \sqrt{\pi D(t-\tau)}}\left(\exp \left(-\frac{(x-\xi)^{2}}{4 D(t-\tau)}\right)-\exp \left(-\frac{(x+\xi)^{2}}{4 D(t-\tau)}\right)\right)
$$

- при граничных условиях вида (6)

$$
G(x, \xi, t-\tau)=\frac{1}{2 \sqrt{\pi D(t-\tau)}}\left(\exp \left(-\frac{(x-\xi)^{2}}{4 D(t-\tau)}\right)+\exp \left(-\frac{(x+\xi)^{2}}{4 D(t-\tau)}\right)\right)
$$

- при граничных условиях вида (7)

$$
\begin{aligned}
& G(x, \xi, t-\tau)=\frac{1}{2 \sqrt{\pi D(t-\tau)}}\left(\exp \left(-\frac{(x-\xi)^{2}}{4 D(t-\tau)}\right)+\exp \left(-\frac{(x+\xi)^{2}}{4 D(t-\tau)}\right)\right)- \\
& -h \exp \left[D h^{2}(t-\tau)+h(x+\xi)\right] \operatorname{erfc}\left(\frac{(x+\xi)}{2 \sqrt{D(t-\tau)}}+h \sqrt{D(t-\tau)}\right)
\end{aligned}
$$

Здесь $\operatorname{erf}(y)=1-\operatorname{erf}(y) ; \operatorname{erf}(y)=\left(\frac{2}{\sqrt{\pi}}\right) \int_{0}^{y} \exp \left(-z^{2}\right) d z-$ функция ошибок.

После выполнения соответствующих подстановок в интегральное представление решения (8) и интегрирования с учетом фильтрующего свойства дельта-функции, были получены аналитические решения рассматриваемых краевых задач.

Решение краевой задачи (1)-(5) имеет вид:

$$
\begin{aligned}
& c(x, t)=\frac{w}{2 D}\left(\operatorname{sgn}\left(x_{0}-x\right)\left(x-x_{0}\right)+\left(x+x_{0}\right)+\left(x-x_{0}\right) \operatorname{erf}\left(\frac{x-x_{0}}{2 \sqrt{D t}}\right)-\left(x+x_{0}\right) \operatorname{erf}\left(\frac{x+x_{0}}{2 \sqrt{D t}}\right)\right)+ \\
& +w \sqrt{\frac{t}{D \pi}}\left(\exp \left(\frac{x x_{0}}{D t}\right)-1\right) \exp \left(-\frac{\left(x+x_{0}\right)^{2}}{4 D t}\right)
\end{aligned}
$$

где $\operatorname{sgn}\left(x_{0}-x\right)=\left\{\begin{array}{c}1, x_{0}>x \\ -1, x_{0}<x\end{array}\right.$.

Решение краевой задачи (1)-(4), (6) имеет вид:

$$
\begin{aligned}
& c(x, t)=\frac{w}{2 D}\left(\operatorname{sgn}\left(x_{0}-x\right)\left(x-x_{0}\right)-\left(x+x_{0}\right)+\left(x-x_{0}\right) \operatorname{erf}\left(\frac{x-x_{0}}{2 \sqrt{D t}}\right)+\left(x+x_{0}\right) \operatorname{erf}\left(\frac{x+x_{0}}{2 \sqrt{D t}}\right)\right)+ \\
& +w \sqrt{\frac{t}{D \pi}}\left(\exp \left(\frac{x x_{0}}{D t}\right)+1\right) \exp \left(-\frac{\left(x+x_{0}\right)^{2}}{4 D t}\right) .
\end{aligned}
$$

Решение краевой задачи (1)-(4), (7) имеет вид: 


$$
\begin{aligned}
& c(x, t)=\frac{w}{2 D}\left(\operatorname{sgn}\left(x_{0}-x\right)\left(x-x_{0}\right)+\left(x-x_{0}\right) \operatorname{erf}\left(\frac{x-x_{0}}{2 \sqrt{D t}}\right)+\right. \\
& \left.+\left(\left(x+x_{0}\right)+\frac{2}{h}\right)\left(1-\operatorname{erf}\left(\frac{x+x_{0}}{2 \sqrt{D t}}\right)\right)\right)+w \sqrt{\frac{t}{D \pi}}\left(\exp \left(\frac{x x_{0}}{D t}\right)-1\right) \exp \left(-\frac{\left(x+x_{0}\right)^{2}}{4 D t}\right)- \\
& -\frac{w}{D h} \exp \left(h\left(D h t+x+x_{0}\right)\right) \operatorname{erfc}\left(\frac{2 D h t+x+x_{0}}{2 \sqrt{D t}}\right) .
\end{aligned}
$$

На рис.2 представлены рассчитанные по формулам (12), (13) и (14) зависимости концентрации дейтерия от глубины в образце $c(x)$. Параметры расчетов: сила тока пучка дейтронов $I=462 \mathrm{HA}$; радиус пучка дейтронов $R=10^{-3} \mathrm{M}$; заряд дейтрона $q_{0}=1,6 \times 10^{-19}$ Кл; коэффициент диффузии дейтерия $D=10^{-12} \mathrm{M}^{2} / \mathrm{c}$; время облучения $t=500 \mathrm{c}$; глубина расположения внутреннего источника $x_{0}=3,69 \times 10^{-6} \mathrm{M}$; коэффициент пропорциональности $h=10^{5}$.

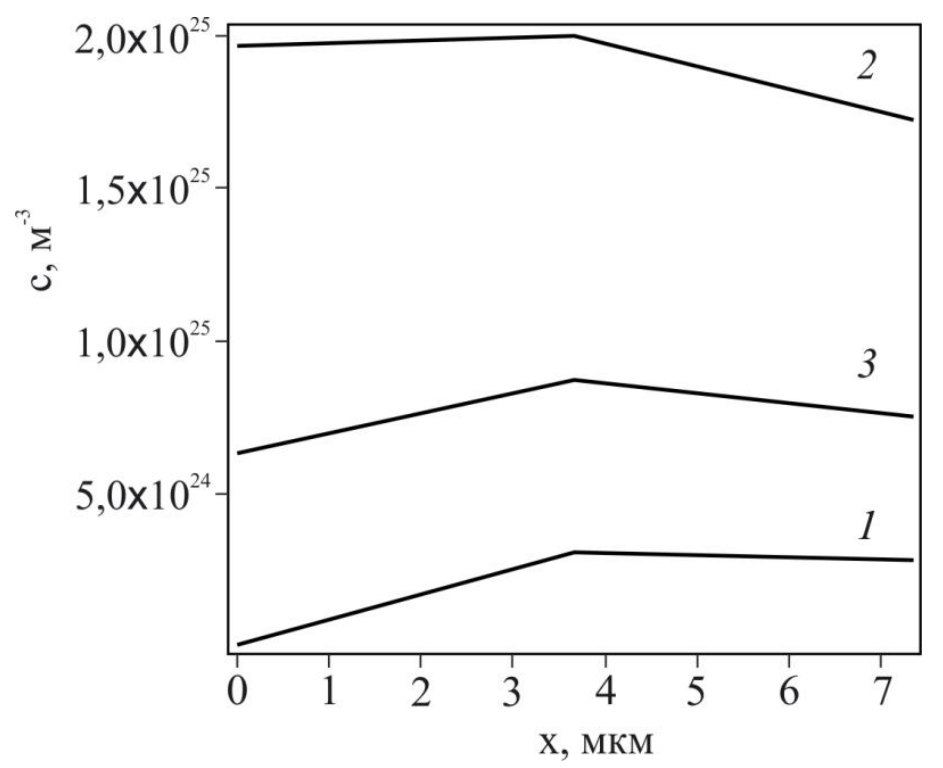

Рис.2. Кривые 1, 2 и 3, соответствующие расчетным формулам (12); (13) и (14)

Разработанный алгоритм является необходимым дополнением к разработанной нами ранее методике NRAOL. Он позволяет провести обработку первичных данных в тех случаях, когда равен нулю поток атомов дейтерия через облучаемую поверхность образца или равна нулю концентрация атомов дейтерия вблизи этой поверхности, а также в промежуточных вариантах. Отметим, что предложенное аналитическое решение содержит экспериментально определяемый в методике NRAOL критерий для корректного выбора граничных условий диффузионной задачи. Им, как видно из рис. 2, является значение концентрации дейтерия вблизи поверхности образца.

\section{2 Распределение ионов кислорода в оксидных наночастицах}

При математической обработке первичных данных ядерного микроанализа по оксидным нанопорошкам принято, что они имеют состав $\mathrm{M}^{16} \mathrm{O}_{\mathrm{x}}{ }^{18} \mathrm{O}_{\mathrm{y}}$, где $\mathrm{M=Ti}$ в случае диоксида титана и $\mathrm{M}=\mathrm{Zr}_{0,82} \mathrm{Y}_{0,18}$ для оксида YSZ10, который содержал 90 мол. \% $\mathrm{ZrO}_{2}$ и 10 мол. \% $\mathrm{Y}_{2} \mathrm{O}_{3}$. Значение индекса $x$ совокупно определялось присутствием атомов кислорода в кристаллической решетке оксида, а также наличием в нанопорошках адсорбированных и хемосорбированных кислородсодержащих молекул. Значение индекса $y$ было практически обусловлено только составом кристаллической решетки оксида. Это связано с очень низким 
содержанием (примерно 0,2\%) атомов ${ }^{18} \mathrm{O}$ в природной смеси изотопов кислорода. Полученные в настоящей работе для диоксида титана линейные зависимости кислородного индекса $y$ от удельной поверхности нанопорошка $S$ (см. рис. 3) были аналогичны таковой для оксида алюминия [7]. Как было показано в этой работе, такие зависимости свидетельствуют о сильном кислородном дефиците в поверхностном атомном слое оксидных наночастиц. Таким образом, не исключено, что формирование на поверхности кристаллической решетки оксида дефицитного по кислороду слоя примерно атомной толщины является общей закономерностью оксидных систем.

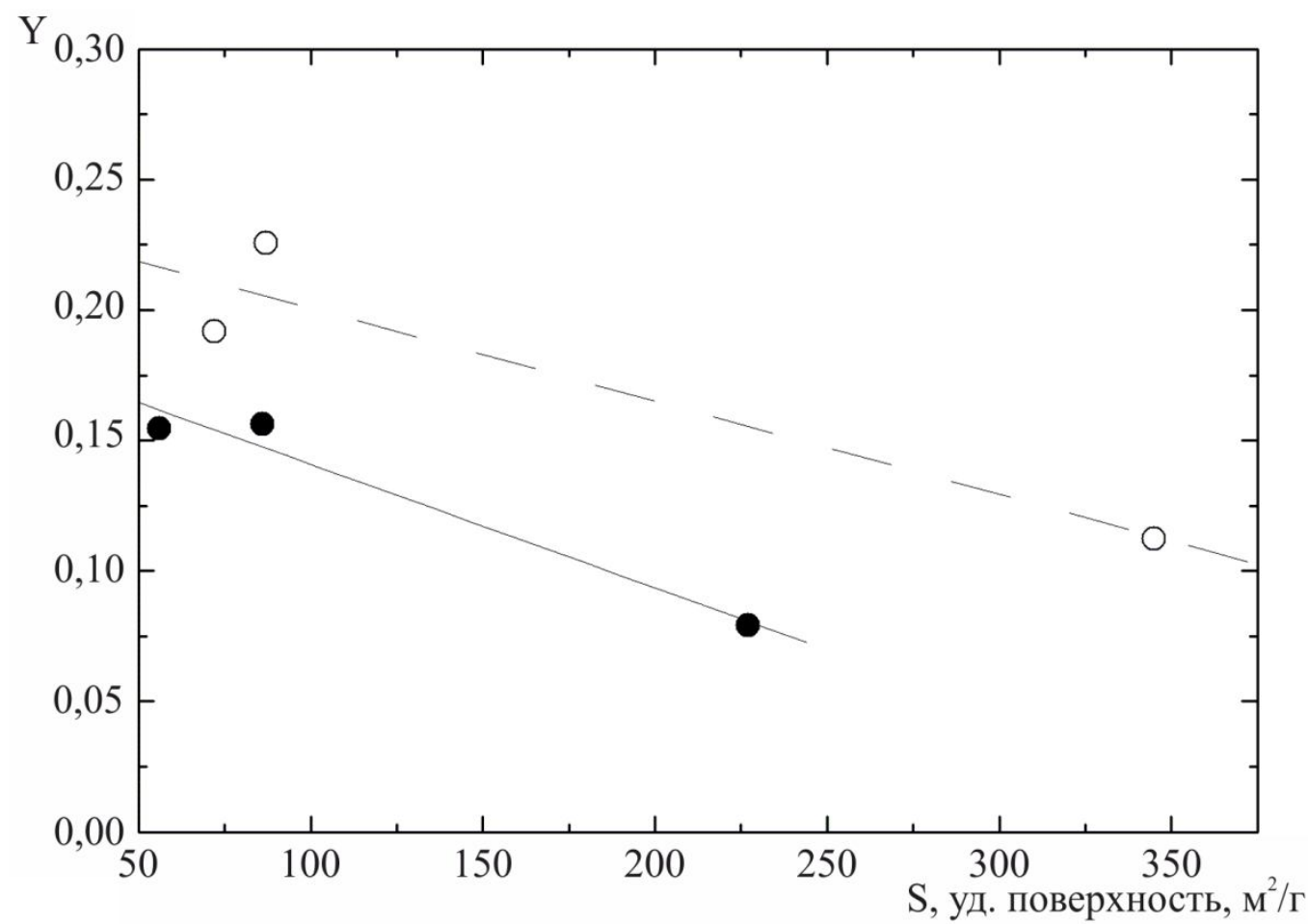

Рис. 3. Зависимости кислородного индекса $y$ от удельной поверхности нанопорошка для диоксида титана $\mathrm{Ti}^{16} \mathrm{O}_{\mathrm{x}}{ }^{18} \mathrm{O}_{\mathrm{y}}$ : открытые кружки - нанопорошки, синтезированные в атмосфере инертных газов; залитые кружки - нанопорошки, синтезированные в атмосфере инертных газов с добавками паров тяжелой воды $\mathrm{D}_{2} \mathrm{O}$

Представленные на рис. 4 данные по $x(S)$ были также аналогичны таковым для оксида алюминия [7], т.е. значение индекса $x$ очень слабо зависело от $S$. Этот результат также, как и зависимость $y(S)$, свидетельствует о значительном кислородном дефиците в поверхностном атомном слое оксидных наночастиц, поскольку при увеличении удельной поверхности нанопорошка возрастает и количество адсорбированных и/или хемосорбированных кислородсодержащих молекул. Для теории и практических приложений важной является информация о термической стабильности кислородного дефицита в кристаллической решетке наночастиц. Для получения данных по этому вопросу мы с помощью NRA проводили измерения концентрации изотопа ${ }^{16} \mathrm{O}$ в нанопорошках оксида $\mathrm{Zr}_{0,82} \mathrm{Y}_{0,18}{ }^{16} \mathrm{O}_{\mathrm{x}}{ }^{18} \mathrm{O}_{\mathrm{y}}$ при повышенной температуре. В нанопорошках этого оксида закономерности $x(S)$ и $y(S)$ были близки к таковым для диоксида титана: при увеличении примерно в таком же интервале удельной поверхности значение индекса $y$ уменьшалось почти в 2 раза, а значение индекса $x$ очень слабо зависело от $S$. Нагрев порошка до $400{ }^{\circ} \mathrm{C}$ в вакууме проводился непосредственно во время измерения концентрации изотопа ${ }^{16} \mathrm{O}$. Такая температура обычно достаточна для удаления адсорбированных молекул воды и других газов и не достаточна для удаления хемосорбированных молекул или термического разложения оксидов. При 
изотермической выдержке при $400{ }^{\circ} \mathrm{C}$ в течение 70 мин наблюдалось уменьшение кислородного индекса $x$ от значения 1,80 до 1,00, т. е. примерно в 2 раза. На качественном уровне эти данные свидетельствуют о том, что кислородный дефицит в кристаллической решетке наночастиц практически одинаков при температуре синтеза и при комнатной температуре.

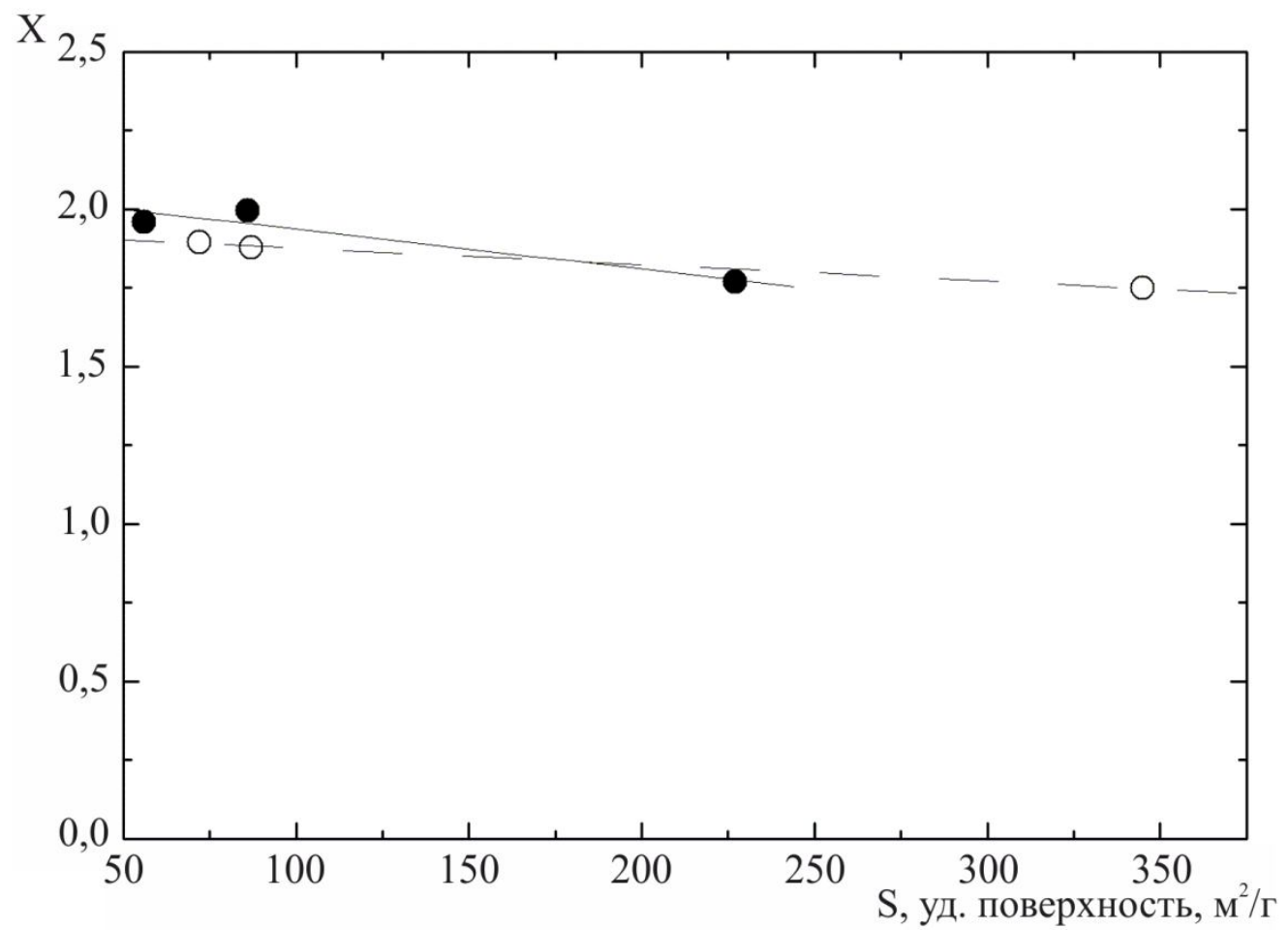

Рис. 4. Зависимости кислородного индекса $x$ от удельной поверхности нанопорошка для диоксида титана $\mathrm{Ti}^{16} \mathrm{O}_{\mathrm{x}}{ }^{18} \mathrm{O}_{\mathrm{y}}$ : открылтые кружки - нанопорошки, синтезированные в атмосфере инертных газов; залитыле кружки - нанопорошки, синтезированные в атмосфере инертных газов с добавками паров тяжелой воды $\mathrm{D}_{2} \mathrm{O}$

Как отмечалось в разд. 1, в настоящей работе была поставлена задача получения данных о влиянии легирования нанопорошков оксида титана на их поверхностную стехиометрию. Из рис. 3 следует, что уменьшение кислородного индекса $y$ при увеличении удельной поверхности наблюдается как при синтезе нанопорошков в атмосфере инертных газов, так и в атмосфере инертных газов с добавками паров тяжелой воды $\mathrm{D}_{2} \mathrm{O}$. Кроме того, с помощью методики NRA не было обнаружено дейтерия в нанопорошках диоксида титана после их синтеза в атмосфере с добавками тяжелой воды. Таким образом, в работе не удалось обеспечить легирование нанопорошков диоксида титана атомами водорода (дейтерия) и получить информацию о влиянии такого допирования на поверхностную стехиометрию оксидных нанопорошков. В то же время из рис. 3 видно, что использование паров воды привело к уменьшению значений кислородных индексов у. Это связано, повидимому, с тривиальным обстоятельством: введение молекул $\mathrm{D}_{2}{ }^{16} \mathrm{O}$ в атмосферу синтеза привело к уменьшению в ней концентрации атомов ${ }^{18} \mathrm{O}$, что обусловило уменьшение значения индекса $y$ в нанопорошках.

\section{4. Заключение}

1. Проведено экспериментальное и теоретическое исследование граничных условий диффузионной задачи при измерении коэффициентов диффузии дейтерия в твердых телах с помощью ускорительной методики ядерного микроанализа в режиме реального времени 
(NRAOL). Установлено, что для металла (никеля) экспериментальные данные удовлетворительно описываются в модели, предполагающей, что атомы дейтерия не выходят из образца при его облучении дейтронами. Для оксида (скандата лантана) это приближение не соответствует экспериментальным данным. Сформулированы начальные и граничные условия диффузионной задачи для методики NRAOL в произвольном случае, они могут быть использованы при исследовании диффузии дейтерия в металлах, оксидах и других материалах.

2. C помощью методики ядерного микроанализа (NRA) проведено исследование поверхностной стехиометрии в нанопорошках диоксида титана и кубического диоксида циркония, стабилизированного иттрием. Кислородная подсистема нанопорошков была обогащена изотопом ${ }^{18} \mathrm{O}$, синтез осуществлялся по технологии лазерного испарения керамической мишени. Установлено, что поверхностный атомный слой кристаллической решетки исследованных нанопорошков характеризуется значительным дефицитом кислорода в сравнении со стехиометрией. Показано, что в случае диоксида циркония, стабилизированного иттрием, кислородный дефицит в кристаллической решетке имеет место как при температуре синтеза, так и при комнатной температуре. Присутствие паров тяжелой по водороду воды в атмосфере при синтезе нанопорошков диоксида титана не приводило к легированию оксида атомами дейтерия.

\section{Благодарность}

Работа выполнена в рамках проекта УрО РАН №15-17-2-10 при частичной поддержке РФФИ (проекты №13-03-00310, №15-03-06617).

\section{Литература}

1. Oxygen Isotope Exchange in Nanocrystal Oxides / Anatoly Fishman, Tatyana Kurennykh, Vladimir Vykhodets, Evgeniya Vykhodets // Advances in Ceramics-Characterization, Raw Materials, Processing, Properties, Degradation and Healing / edited by Costas Sikalidis. - Rejeka : InTech Open Access publisher, 2011. - P. 139-164. - ISBN 978-953-307-504-4.

2. Deuterium migration in titanium during deuteron irradiation observed by proton spectra of the d(d,p)t reaction / Hiroshi Kudo, Yasuo Kosaku, Yuji Ando, Masayuki Hiraga, Tsutomu Sekine // Journal of Nuclear Materials. - 1998. - Vols 258-263, p. 1. - P. 622-627.

3. Deuterium diffusion in proton conductors $\mathrm{La}_{0.9} \mathrm{Sr}_{0.1} \mathrm{ScO}_{3-\delta}$ and $\mathrm{BaZr}_{0.9} \mathrm{Y}_{0.1} \mathrm{O}_{3-\delta}$ at room temperature / V. B. Vykhodets, T. E. Kurennykh, O. A. Nefedova, V. P. Gorelov, A. Yu. Stroeva, V. B. Balakireva, E. V. Vykhodets, S. I. Obukhov // Solid State Ionics. - 2014. - Vol. 263. - P. 152-156. - DOI: 10.1016/j.ssi.2014.06.003.

4. Kidson G. V. The diffusion of H, D, and T in solid metals // Diffusion in Solid Metals and Alloys / edited by H. Mehrer. - Berlin : Landolt-Bornstein, Springer-Verlag, 1990. - ISBN 354050-886-4. - Vol. III-26. - P. 504-556.

5. Völk1 J., Alefeld G. Hydrogen Diffusion in Metals // Diffusion in Solids: Recent Developments / edited by A. S. Nowick, J. J. Burton. - New York : Academic Press, 1975. - P. 231-302. - ISBN 0-12-522660-8.

6. Diffusion in Condensed Matter: Methods, Materials, Models / ed. by Paul Heitjans, Jörg Kärger. - Berlin-Heidelberg : Springer, 2005. - 965 p. - ISBN 3-540-20043-6.

7. Extreme deviations from stoichiometry in alumina nanopowders / V. B. Vykhodets, E. Jarvis, T. E. Kurennykh, A. E. Davletshin, S. I. Obukhov, I. V. Beketov, O. M. Samatov, A. I. Medvedev // Surface Science. - 2014. - Vol. 630. - P. 182-186. - DOI: 10.1016/j.susc.2014.08.009.

8. Jarvis E. A. A., Carter E. A. Metallic Character of the $\mathrm{Al}_{2} \mathrm{O}_{3}(0001)-(\sqrt{31} \times \sqrt{31}) \mathrm{R} \pm 9^{\circ}$ Surface Reconstruction // J. Phys. Chem. B - 2001. - Vol. 105, iss 18. - P. 4045-4052. DOI: $10.1021 / \mathrm{jp} 003587 \mathrm{c}$. 
open-access journal

9. Wang X. - G., Chaka A., Scheffer M. Effect of the Environment on $\alpha-\mathrm{Al}_{2} \mathrm{O}_{3}$ (0001) Surface Structures // Physical Review Letters. - 2000. - Vol. 84. - P. 3650-3653.

10. Crank J. The mathematics of diffusion. - London : Oxford University Press, 1975. - 414 p. - ISBN 0-19-853344-6.

11. Nellis G., Klein S. Heat Transfer. - Cambridge : Cambridge University Press, 2009. 1107 p. - ISBN 978-0-521-88107-4.

12. Карташов Э. М. Аналитические методы в теории теплопроводности твердых тел. М. : Высш. шк., 2001. - 550 с. - ISBN 5-06-004091-7. 